Thomas's Hospital, where she rapidly recovered. Nothing abnormal was detected on examination with the $\mathrm{X}$ rays. The weapon was a small Browning repeating pistol, firing a bullet the size of the one shown in the skiagram.

The man continued in a very low state after admission, and it was found that he was unable to move his lower limbs. He had retention of urine. There was complete anæsthesia below the waist and entire loss of reflexes. The bullet was seen with the $\mathrm{X}$ rays, but this examination was unsatisfactory. As the condition of the man prevented full exposure it could not be definitely localised. It was, however, lying to the right of the middle line in the spinal region. As there was still a rery rapid pulse with some hæmorrhage from the wound, he was placed under an anæsthetic in the erening. The wound was opened up and the lung examined. There had been very little hremorrhage into the pleura; the wound of the lung could be easily seen, but the lung was collapsed and the pleura full of air. The pericardium which was immediately to the right of the wound was uninjured. The edges of the wound in the intercostal space were pared and the opening closed with sutures. There was a gradual improvement in the condition of the chest and the general state of the patient, but none in that of the paraplegia. Dr. A. D. Reid then carefully examined the patient with the $X$ rays, and gave it as his opinion that the bullet was lying in the spinal canal at the level of the seventh dorsal vertebra (see illustration). On the chance that its removal might be of benefit in the relief of pressure, laminectomy was performed on Nor. 2nd and the bullet was remored. It was lying between the dura mater and the rertebra to the right of the middle line. No irregularity of the bone could be felt or seen. The bullet was not in any way fixed, and was easily lifted out when the cord had been retracted to the left. It had not lost much metal, being but slightly flattened at the tip as if it had been deflected by bone or something of a hard nature. The cord was evidently much bruised. Pulsation was visible to the level at which the bullet was lying, but not below that. The patient stood the operation rery well and the wound healed satisfactorily. He thought that sensation improved in both legs, but the paralysis did not get any better. Retention with orerflow was only prevented by catheterism, and cystitis dereloped, from the effects of which he died on Nov.21st at 9 P.M.

No post-mortem examination was permitted and a verdict of suicide was returned by the coroner. The radiograph, which is an excellent one, enabled Dr. Reid to say that the bullet was lying in the spinal canal, at the level of the seventh dorsal vertebra, and it was exactly as he stated. As there was no trace of bone injury or splashes of lead on the bones, did the bullet enter the spinal canal by the intervertebral foramen and turn up? To gain this point did it travel along the inner aspect of the ribs, or pass direct to the foramen which was penetrated?

Questions which present themselves for solution are, Did the man shoot the woman and himself, as he stated? If the man shot himself, how was it the bullet was lying so much to the right of the wound of entrance that it was removed from the right side of the spinal canal? Would any healthy man lying in bed naturally place a revolver in such a position as that in which this one was found? Could a man who had received such a severe mental and physical shock place the weapon so? Is it probable? The shortness of the interval between the two reports makes it unlikely that the weapon. changed hands.

These are the questions which would naturally present themselves in any medico-legal examination of the facts, but such examination was considered unnecessary in view of accompanying circumstances and the man's confession.

Harley-street, W.

\section{NASOPHARYNGEAL AND CERVICAL GLANDULAR TUBERCULOSIS IN CHILDREN.}

By A. DINGWALL FORDYCE, M.D. EDIN., F.R.C.P. EDIN.,

EXTRa PHYSICIAN, RoYaL HOSPITAL FOR SICK CHIIDREN, EDINBURGH; AND

E. W. SCOTT CARMICHAEL, M.B. EDIN., F.R.C.S. EDIN.,

ASSISTANT SURGEON, ROYAL INETRMARY, EDINBURGH.

Physicians and surgeons, more particularly those connected with a hospital for children, are confronted daily with cases of this nature. Our views on the subject are based on the experience gained by the treatment and observation of a large number of hospital cases, and a not inconsiderable number of private patients. We consider the subject from the following points of view: (1) Etiology and pathology; (2) clinical course; (3) prophylaxis; and (4) treatment.

\section{Etiology.}

In children under 1 year. During the early months of the first year of life tuberculosis is a comparatively rare disease, but in cases in which it occurs it is often in relation to the structures of the middle ear. In children under 6 months of age middle ear and mastoid tuberculosis is relatively common. It may be suspected from three clinical signs and symptoms: (1) a watery discharge from the midale ear ; (2) a soft; boggy, painless swelling over the mastoid process; and (3) facial paralysis. Facial paralysis coming on in a child under 6 months of age, unaccompanied often by discharge from the external anditory meatus, or by perforation of the tympanic membrane, or by external evidence of mastoid disease, is almost a pathognomonic sign of extensive tuberculous disease of the Eustachian tube, middle ear, and mastoid process. This fact is very important. A soft swelling, usually painless, which is somewhat diffuse over the mastoid process and causes the pinna to bulge forward, is equally suggestive of tuberculosis. In such a case there is often no discharge from the external auditory meatus. We have never seen such signs present in a child of this age who was solely fed at the breast. We are bound therefore to correlate the occurrence of such early tuberculous infection with the practice of artificial feeding, and, more particularly, with feeding with cow's milk. There is a marked predilection for the infection to affect the middle ear and the temporal bone, and it seems to us that this is explained by aspiration up the Eustachian tubes during the act of sucking. It is certain that as the child gets past the age of sucking, mastoid and middle-ear infection become less common, and the disease attacks other regions" of the nasopharynx. In these cases the tuberculous infection is often very acute and virulent. If, however, the disease remains limited to the mastoid process and does 
not spread either to the cervical glands or cranial cavity, the prognosis is a good one. We would therefore draw attention to the facts that tuberculosis affecting the nasopharynx in children under 1 year of age, and especially those under 6 months, has a strong predilection to spread up the Eustachian tube, and that it invariably occurs in children fed with cow's milk.

Large hospital experience of glandular tuberculosis in children over 1 year has proved to us that one gland or set of glands in the neck is more liable than any other to be the site of infection. This gland is situated in a definite anatomical triangle in the upper part of the neck. It lies immediately below the posterior belly of the digastric, and between the internal jugular and common facial veins. It is the central point of conveyance of a large number of lymphatics. These are, indirectly, the auricular, the facial, and the submaxillary, but above all it is the gland which receives directly the lymphatics from that part of the nasopharynx which includes the tonsil. has also intimate connexions with two other sets of glands: (1) half a dozen round the spinal accessory nerve as it enters the sterno-mastoid muscle; and (2) the carotid set, passing down in relation to the carotid sheath. It is important to understand these anatomical facts in order to realise the course of tuberculous infection in this region of the body. Why should this gland (jugulodigastric) be more liable to infection by the tubercle bacillus than any other in the body? We must realise that it is almost invariably a secondary infection. Where, then, is the primary focus? Some years ago one of us (E. W. S. C.) examined the pharyngeal tonsils in a series of cases of glandular tuberculosis. Casual sections were made of the tonsil on the side on which the glands were proved to be tuberculous after operation. In nearly 20 per cent. of cases the tonsil was found to be the site of tuberculous infection. It was only natural to suppose that a more careful examination by means of serial sections of such tonsils would show a still greater percentage of tonsillar infection, and such has proved to be the case from the recent work of Stiles and Mitchell. Tuberculous infection of the tonsil, therefore, is now known to be extremely common, and we must refer to its pathology in order to understand the course of events in the common form of the disease.

\section{Pathology.}

Primary infection: tonsillar tuberculosis.-Tuberculous infection of the tonsil does not cause ulceration of its surface: we have never seen a case of tuberculous ulceration even in tonsils which were the seat of advanced tuberculosis. In our experience tonsils which are the seat of extensive tuberculous infection are not hypertrophied tonsils, but are, on the contrary, often small, ragged, and atrophic. Tonsillar tuberculosis is chronic in type and leads to fibrosis and shrinkage of the tonsil. It rarely causes caseation to such a degree as to lead to breaking down of the tonsil. In the early stages it begins in the form of ore or two giant cell systems round the bottom of the tonsillar crypts. These gradually extend into the substance of the tonsil until the whole or part of the gland has become invaded by the disease. It is probable that the tonsil is often tuberculous months or even years before the disease spreads beyond its substance. When the disease reaches the outer boundary of the tonsil it sets up a peritonsillitis, which is often somewhat acute in character. It is not perhaps going too far to say that many of the cases of acute tonsillitis so-called, which are ultimately found to be associated with tuberculosis of the corresponding glands, are really peritonsillar infections, and due to the spread of the disease beyond the tonsil. We have seen numerous instances where an attack of acute tonsillitis has led to the discovery of an advanced glandular tuberculosis.

Secondary infection : glandular tuberculosis.Tuberculous infection in a gland begins as isolated foci which are often of different ages and stages of development. On examining such a gland removed at operation these multiple foci are seen distinctly, and consist of a caseating or calcareous centre with a surrounding capsule of fibrous stroma. When the tuberculous condition is progressive these foci continue to grow, and sooner or later one reaches the surface of the gland, involves its capsule, and sets up an inflammatory condition around the gland (periadenitis). This may follow one of two courses: (1) The periglandular tissue becomes congested and œdematous. This leads to extensive swelling which persists for some weeks and finally subsides. (2) This process does not resolve but passes on to the formation of an abscess outside the gland, which ultimately makes its way to the skin surface. In a large number of cases the periadenitis subsides, but another similar attack is liable to occur, and frequently recurring attacks end sooner or later in abscess formation. It is interesting to note that these recurrent attacks of periadenitis are not due to mixed or septic infection, and we would emphasise the resemblance in this respect between periadenitis and peritonsillitis already referred to. One of us (E. W. S. C.) has examined the pus and tissues from these cases of periadenitis on several occasions, and has failed to demonstrate the presence of any pyogenic organisms.

\section{Clinical Course.}

\section{The following are examples of cases.}

CASE 1.-A female aged 6 years. She was a healthy baby of healthy parents, and was brought up on the bottle and given large quantities of milk and cereals early. Purely pap feeding was continued through the second year, and no animal food was given other than milk or an occasional egg. Digestion and health were generally fairly good. When $2 \frac{1}{2}$ years old she suffered from acute tonsillitis. A throatswab showed streptococci. The cervical glands on one side of the neck rapidly enlarged and became matted by periadenitis. In the course of about 10 days the throat condition had largely subsided, but in the neck a lump of the size of a small orange remained. In spite of general treatment glandular enlargement persisted. The tonsils were not markedly enlarged, but were ragged and unhealthylooking. Operation on the tonsils or on the glands was refused. The child continued in fairly good though uncertain general health. An excessive carbohydrate diet was persisted in and the mass of glands gradually softened. When aged 6 years, along with pharyngeal catarrh, the enlargement rapidly increased and a large abscess was developed which threatened to rupture externally. On operation being performed extensive tonsillar tuberculosis, with a large abscess and numerous broken-down tuberculous glands in the neck, was found.

CASE 2.-A female aged 4 years. She was taken ill suddenly with influenza. She had a high, swinging temperature, sore throat, and greatly enlarged tonsils, with numerous white patches on the surface. A throat swab showed streptococci. On recovery from the acute attack the tonsils and adenoids were removed and found to be tuberculous. Six months later as a mass of glands remained on one side of the neck it was removed and found to be tuberculous. 
CASE 3.-A male aged 18 months. He was a healthy baby at birth. He was brought up on the bottle and fed largely on patent foods and medicated milk. He had constant digestive difficulties, and developed slight rickets, with distinct symptoms of the presence of adenoids and unhealthylooking ragged tonsils. At the age of 14 months he developed acute tonsillitis with great enlargement and matting of glands on one side of the neck. Two months later excision of adenoids and tonsils showed tuberculous infection throughout.

Such are common and typical cases of apparent acute outset. But the occurrence of sore-throat and acute and marked glandular enlargement is no evidence of the period of onset of the tuberculous infection, and in most cases the tonsil has probably been the seat of a latent tuberculous infection for some considerable time previously. Generally speaking, the younger the child affected the more acute is the course of the disease. When the disease runs throughout a chronic course we take it that the course is nevertheless similar to that in an acute case. The difference here is that the conditions of peritonsillitis and periadenitis are slighter and may not be recognised clinically. The first evidence of the presence of disease is then the appearance of a single mobile gland in the side of the neck. This gland may lie latent for years, and, beyond a slight variation in size from time to time and an occasional slight attack of periadenitis, it does not attract much attention. A focus in its substance, however, may at any time become active and lead to the formation of an abscess.

\section{Prophylaxis.}

Tuberculous infection of the tonsils and cervical glands occurs in cases in which, apparently, every rational method of procedure has been adopted. Often, however, it is far otherwise. It is not our object at present to direct attention to the infecting agent-the tubercle bacillus. Suffice it to say that, however large or however small a proportion of cases it may be which are infected by the bacillus of bovine origin, it is always wise to give only scalded milk to a baby during the first two years of life. Prevention otherwise naturally consists in the maintenance of good general nutrition and of a healthy condition of oral and nasopharyngeal tissues. For the attainment of these ends two factors are of great importance-diet and oral hygiene.

The foundation of tuberculous disease of the cervical glands is over and over again laid in the manner of infant feeding. Unsatisfactory digestion, imperfect nutrition, eructation, vomiting, and consequent nasopharyngeal catarrh ensure a suitable nidus for infection. The advantage to a baby of receiving his mother's milk is too obvious to need more than remark. If he is not suckled he should receive during the first two years of life no cow's milk which has not been scalded. He should be fed on fixed quantities at definite intervals. This is the most important rule in infant feeding, and the one which is generally most necessary to emphasise. Within reason the actual composition of the food for a healthy baby is much less important. Cow's milk, condensed milk, patent foods -babies thrive well on all or any of these. Some are better than others, but most healthy babies would thrive on any if judiciously handled. But irregularity and uncertainty in time and amount of feeding are always dangerous, and usually have disastrous results. Irregularity of feeding times nearly always means feeding at too short intervals, and uncertainty of the requisite amount at each feed usually ends in overfeeding. In both cases regurgitation and often vomiting tend to occur. Catarrh of the pharynx is set up, and in association with disordered digestion and its effects on nutrition, a favourable locus is provided for infection. For the same reason cereals or patent foods containing unaltered starch should be avoided as a rule during the first six months of life.

The commencement of primary dentition-the latter half of the first year-is a period of great importance from the dietetic point of view. Starch now is a valuable element in the diet, the chewing and gnawing of hard food has to be encouraged, and simple animal food, such as gravy, clear soup, and lightly boiled egg, has to be gradually included in the diet sheet.

We would emphasise the importance of accustoming the baby to gnaw a tough crust or a thick, strong bone. Oral hygiene largely consists in facilitating the cleansing of the mouth by saliva, and by no means can this be better attained than by such gnawing and chewing. During the second. year considerable variety of food is good, hard food is useful, and meat becomes an important element of the diet.

Here we would lay stress on a prevalent misconception regarding the value of meat in the diet of young children. Constantly we see otherwise healthy children suffering from indigestion due to overfeeding with bread, porridge, potatoes, biscuits, cakes, sweets. Comparatively rarely do we see indigestion due to excessive meat-eating. Most certainly a large amount of meat in the diet of a healthy young child is likely to cause digestive trouble. But the point is that an adequate supply of flesh meat and fish in the daily diet is not only beneficial, but is, as a rule, necessary for the maintenance of health. During the first half of the second year the child should get this animal food in the form of egg, bacon, or fish. Thereafter he should be given daily at dinner a little chicken, rabbit, or butcher's meat. We consider that deficiency of animal food-in the shape of meat, fish, and eggs-in the diet, along with the common coexistent carbohydrate dyspepsia, is a widespread and important underlying factor in the development of various infections, and is more particularly one of the primary causes of the vast amount of cervical glandular tuberculosis.

\section{Treatment.}

As has been indicated, the onset of obvious enlargement of the glands is often sudden. The child is seized with a general illness of the nature of a "cold" or influenza, there is acute pharyngeal catarrh and often marked tonsillitis, and coincidently there is glandular swelling in the neck. With ordinary mild antiseptic local treatment the acute condition in the throat subsides in the course of a few days, but the glandular enlargement remains. Von Pirquet's reaction is strongly positive. There is a mass on one side of the neck of the size of a small orange and adherent to the surrounding tissues. The mass is firm and at first tender on manipulation. On the other side of the neck there are usually one or two small firm discrete glands to be made out on careful palpation. Under no circumstances should operative interference be carried out when peritonsillitis or periadenitis is present. Surgical interference is only indicated after or between such attacks.

The first and obvious procedure is to seek to 
remove the acute periadenitis by means of boric poultices or ichthyol (20 per cent.) applications locally, and to paint the throat with a mild antiseptic (boroglyceride). As the condition improves the tenderness disappear's, the glands appear firmer and more readily movable, and the mass shrinks to some extent. During this period no other routine medical treatment should be employed and tuberculin should certainly be avoided. The general health of the child, however, requires careful attention. Often it is quite good, but sometimes there are want of appetite, lassitude, and various signs of disturbed metabolism. Usually, however, there is no swinging temperature. Four to six weeks after the onset of the condition the tonsils and adenoid tissue should be removed. This should be done in every case, whether there is apparent enlargement or not.

Following on this operation the child should be ordered a change of air for about two months. If it is summer-time and the weather warm the sea coast is suitable, but as a rule an inland part of the country at some height is better. During this time the child is in the open air as much as possible, is given plenty of simple food at regular intervals, and in particular receives a sufficiency of animal food. As a matter of routine the administration of tuberculin is inadvisable. If the child can be given all the stimulating and invigorating advantages which accrue from a suitable change of air and scene and a well-regulated diet, tuberculin gives nothing more. If, on the other hand, the best environment in all respects cannot be obtained, tuberculin is of value. Tuberculin T.R. is given in small doses every tenth day. For a child of 2 years one begins with a dose of $1 / 8000$ th of a milligramme and increases to $1 / 6000$ th ; for a child of more than 5 years one begins with a dose of 1/6000th and increases to $1 / 4000$ th. The attempt is to avoid any general reaction. At the end of two months-i.e., between three or four months after the onset of the acute condition-the question whether or no operation is advisable should be decided. In all cases, however, where before this time there has been evidence of active disease as shown by recurring attacks of periadenitis, operation should be advised. This is particularly the case when the tonsils hare been found to be extensively diseased.

The younger the patient the more active the disease as a rule, and the greater the need for surgical interference. If there is a definite history of family predisposition to tubercle infection, and, more particularly, if a member of the household suffers from tuberculosis, and if the glands have not distinctly shrunk, they should be removed at once, irrespective of the general condition of the child. The probability in such cases is that sooner or later they will break down. If there is no such history, however, if the general condition of the patient is not quite satisfactory, and if the glands show no distinct signs of shrinking, or if the disease is spreading to the spinal accessory or carotid set of glands, or if there are signs of abscess formation-again operation should be advised at once. If the general condition is good and the glands are distinctly smaller, operation is not advisable, and there is good ground for the hope that, under a careful regimen, the condition will undergo a natural cure. If the general condition is not quite satisfactory, but the glands are rather smaller and firmer, or, again, if the general condition is good but the glands are not distinctly smaller, a course of tuberculin should be employed for a further period of at least two months. If at the end of six months from the acute onset, in the one case the general condition is still unsatisfactory, or in the other the glands are still markedly enlarged, they should be removed. In cases, chronic from the commencement, where a gland persists for mole than a year and tends to get lar'ger, its removal is desirable.

\section{MODERN TREATMENT OF LACRYMAL OBSTRUCTION.}

BY D. LEIGHTON DAVIES, M.D., M.S. LOND., F.R.C.S. ENG.,

ASSISTANT LECTURER AND DEMONSTRATOR OF ANATOMY, UNIVERSITY COLLEGE OF SOUTH WALES AND MONMOUTHSHIRE; ASSISTAN' OPHTHALMIC SURGEON TO THE CARDIFF INFIRYARY.

THE recent discussion at the annual meeting of the British Medical Association on the treatment of lacrymal stricture appears to have been of little practical value, for it has left us in much the same state as we were in before. My object in writing this paper is to appeal to ophthalmic surgeons for a wider trial of rational lines of treatment in cases of lacrymal duct obstruction.

For many years ophthalmic surgeons hare been satisfied with the old-established method of probing; but of late years there has been a growing tendency to resort to other methods. The large number of lacrymal sacs that are now excised annually bears eloquent testimony to the unsatisfactory results from probing. Undoubtedly probing does at times meet with brilliant success, but such cases cannot be frequent, else why this wholesale excision of sacs? The reasons for the failure of the various probing methods are numerous. Amongst them is the tendency for the stricture to close, necessitating repassing of probes at intervals for rears, or at any rate until the patient gets tired of the treatment. Let us think for a moment what is likely to happen in the passage of a full-sized probe. A certain amount of force, more or less, is used, and this must in all probability actually denude the lacrymal canal of its mucous membrane at the point of greatest pressure. We cannot even be certain that the probe does not pass through a tear in the mucous membrane and for the rest of its course pass down between the mucous membrane and the bony wall of the canal.

Compare the conditions to be found in a case of urethral stricture. There we know that fibrous tissue replaces the ordinary mucous membrane, and after the relief of a tight stricture by the passage of bougies it is recognised by surgeons that the cure is not permanent, but the patients are usually instructed to present themselves at regular intervals for the passage of bougies. Neglect of this advice sooner or later results in a recontraction of the stricture. How can we expect the lacrymal canal to differ in its course and progress when treated on the same lines?

The use of styles following probing has had a good many advocates. They do not always relieve, in many cases they are a source of discomfort, and in not a few cases have patients presented themselves at the hospital with marked cellulitis of the tissues round the orbit due to a forgotten style, which in some cases had been there for years and was extracted with difficulty.

I now pass on to the question of the extirpation of the sac, the various methods for which are numerous and need not be discussed. In farour 